\title{
Context Aware PAA for Mobile Shopping and Payment
}

\author{
Richa Sharma \\ Department of Information Technology \\ VES Institute of Technology \\ Chembur, Mumbai, India
}

\author{
Shalu Chopra \\ Head of Department, Information Technology \\ VES Institute of Technology \\ Chembur, Mumbai, India
}

\begin{abstract}
As mobile phones become cheaper and technically advanced more and more people are adopting m-commerce. However shopping from wide range of options available online and offline is often a challenging job. This paper proposes iShopper an intelligent shopping agent which aids user in shopping hard good product proactively. The aim of the system is to avail the user best product and payment option automatically. Context aware Muti-Agent architecture is adopted for negotiations between the buyer and the seller. Decision making is done by inferring knowledge from the semantic relationship defined in ontology and on the basis of predefined JESS rules.
\end{abstract}

\section{General Terms}

Multi-agent, Context Aware, Ontology

\section{Keywords}

PAA, iShopper

\section{INTRODUCTION}

As phones become handy and smart, their roles in our lives also become wider and large. The unprecedented growth of communication technologies has created a great impact on our lives and has changed our ways of executing task. Traditional brick and mortar shopping culture has been overtaken by mshopping wave. For example, previously, buying a camera would require the customer to personally visit various shops, compare the product features and prices offered by different sellers. However now with so many shopping portals available, buyer can simple login to one of them and see all the options available at a single click. Things have become even more simplified with recommendation system. The portals can now recommend the user various camera options that suit their requirements and thus reduce their searching time. Such Systems can be further enhanced by imparting intelligence in them so that they can select the best possible camera option for the user using his contextual data and automatically lead them to best possible payment option after negotiating with various sellers. For instance if the user wants to buy a Sony's amateur digital camera ranging between INR 5000 to 10000 ,the system would pick the best buy for him. Further it also selects the best payment option and the dealer providing it.

In case if the buyer is travelling and wants to know the best buying option the system can proactively take into consideration buyers needs and suggest him/her the best option available. Contextual information and agent technology helps in achieving pro-activity in a system. The Context is any information through which the status of entities can be explained. An Entity is an individual, a location or any object related to the interactions between the user and the application. In Ubiquitous Environments, context can be explained as a collection of personal information and user's needs, his present activities plus information by considering environmental and physical conditions [6].Context information effective in physical goods consists of following parts:

- Context information effective in goods price selection

- Context information effective in selection of payment

- Context information effective in Sellers preference

Context information effective in goods price selection includes free transportation fares or discount offered by the seller, packing cost, tax and customs duties, seller's credit, delivery duration, offering discounts for shipping multiple items in one order, etc.

Influential context information which is crucial in selecting method of payment includes order amount, user's preferences e.g. payer anonymity, the period of transaction (micropayment should be fast), involvement of financial institutions and the amount of money they get as commission.

Seller's preferences for example offering discount if payment is done in special manner, the status of credit card, the capabilities that mobile network operator offers, security requirements, device specifications, payment time (per-paid, pay-now system, post payment), the characteristics of issuer bank and acquirer bank and so on [1].

Finally to deal with different buyers diverse preferences, personalization is needed. For example in camera purchase some buyers may be prioritizing on price, others on pixels and some others might feel warranty services as most important factor. PAA is implemented to hide complexities and provide personalized services to the user [7]-[13]. It captures user requirements and contextual data and sends this information to multi- agent server and gets the selected product from the server. The server has Processing agent which uses semantic relationship described in ontology and rule based inference engine to select a single product best suited for user purpose. It also negotiates with seller agents and selects the best seller option as per user's payment preferences. Finally the Payment Manager performs the necessary payment related operations.

In this paper, iShopper a personal assistant agent has been proposed to hide all the complexities of product selection and mobile payment services from users and automatically looks after the activities related to the personal mobile payment. In continuation, section 2 discusses related works, section 3 discusses System architecture, section 4 talks about system evaluation and testing and finally section 5 talks about conclusion and future work.

\section{RELATED WORK}

AT\&T Bell Laboratories came up with shopping assistant guide which guides the shoppers through the store, provide 
details of items, and help locate items, point out items on sale and do a comparative price analysis. The store maintains customer profiles. Customers are divided into two classes: regular customers who shop anonymously without profiles in store, and store customers who signed up with a store and will get additional discounts in exchange for sacrificing their privacy [2].

Lukkari and Korhonen have established a smart restaurant with the capability of mobile payment in a context aware environment. The Web service of this smart restaurant is installed on a host and then users are connected to this Web service through their mobile devices and they can determine the time of their food delivery. In this restaurant, payment relies on the time and location [1], [3].

Borman and Flake have shown a context aware service discovery system for advice of charge service, for example when you go on a trip to abroad, you can see different mobile network operators which are different in their fees. By using context aware advice of charge service the rating and charging engine introduces the best and most useful mobile network operator to the user. The user can easily send their preferences which can be the most inexpensive mobile network operator or a service with a higher band width to the rating and charging engine. Then on the basis of the user's preferences and other context information like time, location and so on. The system sends the user the best mobile network operator [4].

Previous research suggests models using context aware multiagent systems but so far not much research has been done for a context aware PAA which proactively selects the best product deal as well as mobile payment option for the user on a single click.

\section{SYSTEM ARCHITECTURE}

Overview of system architecture is show in fig 1. The system is divided in three main parts- Application Layer, Integration/Core Layer and the Data Layer.

\subsection{Application Layer}

The Application layer as shown in fig 1 consists of Authentication/ Registration, PAA and Android Apps which are installed on Users Android Phones.

\subsubsection{Authentication/Registration}

This Block handles registration and authentication process of the system. First time users register themselves by filling the registration form. On its completion a random password is and generated sent to users email id. User can then login using his email id and the password given. In case if the user forgets his password a new password is created and mailed on his email id. User can also change their existing password. The process is described in Fig. All passwords are stored in encrypted form in backend.

\subsubsection{Personal Assistant Agent (PAA):}

The primary role of PAA installed on the Android Phone is to hide the complexity of the system from the user and provide products and services with minimal level of input and interaction. PAA collects user requirements and preferences and sends it across to the next layer (Integration / Core layer) located in the main server through Wi-Fi/gprs.

\subsubsection{Android Apps}

Consist of various android apps installed on smart phone.

The implementation was done using Jade-Leap, JDK 1.7 and Android UI Components with Java as the programming language.

\subsection{Integration/Core Layer}

The Integration/Core layer is the key and most important part of the iShopper System. It consists of multiple subsystems like the Processing Manager, Negotiating Manager and Payment manager. These subsystems interact seamlessly with each other and other layers. Each of these subsystems has their own set of agents which are explained in details below. The implementation was done using Jade, JDK 1.7 and Java as the programming language. The Domain ontology was accessed using Jena/Sparql and Jess was used as the Inference engine. The MySQL database was accessed and updated using JDBC connectivity

\subsubsection{Processing Manager}

The Processing Manager is the central part of the iShopper application residing on the Server. It executes most difficult task within the Application and is also responsible for interacting with other layers and agents of the System. The internal structure of Processing Manager is shown in Fig 2.

Processing Manager receives User request and contextual data from the PAA through wifi/GPRS. Its task is to select best product for the User from the repository. It also invokes Negotiating Manger to get the best Seller for the selected product based on User preferences and contextual information.

The context modelling and relationship of products and its properties is done using Web Ontology Language which is accessed Using Jena/SPARQL. The output from the domain ontology acts as input to both Pre-Processing rule and PostProcessing Jess rule which are executed using Jess engine. 

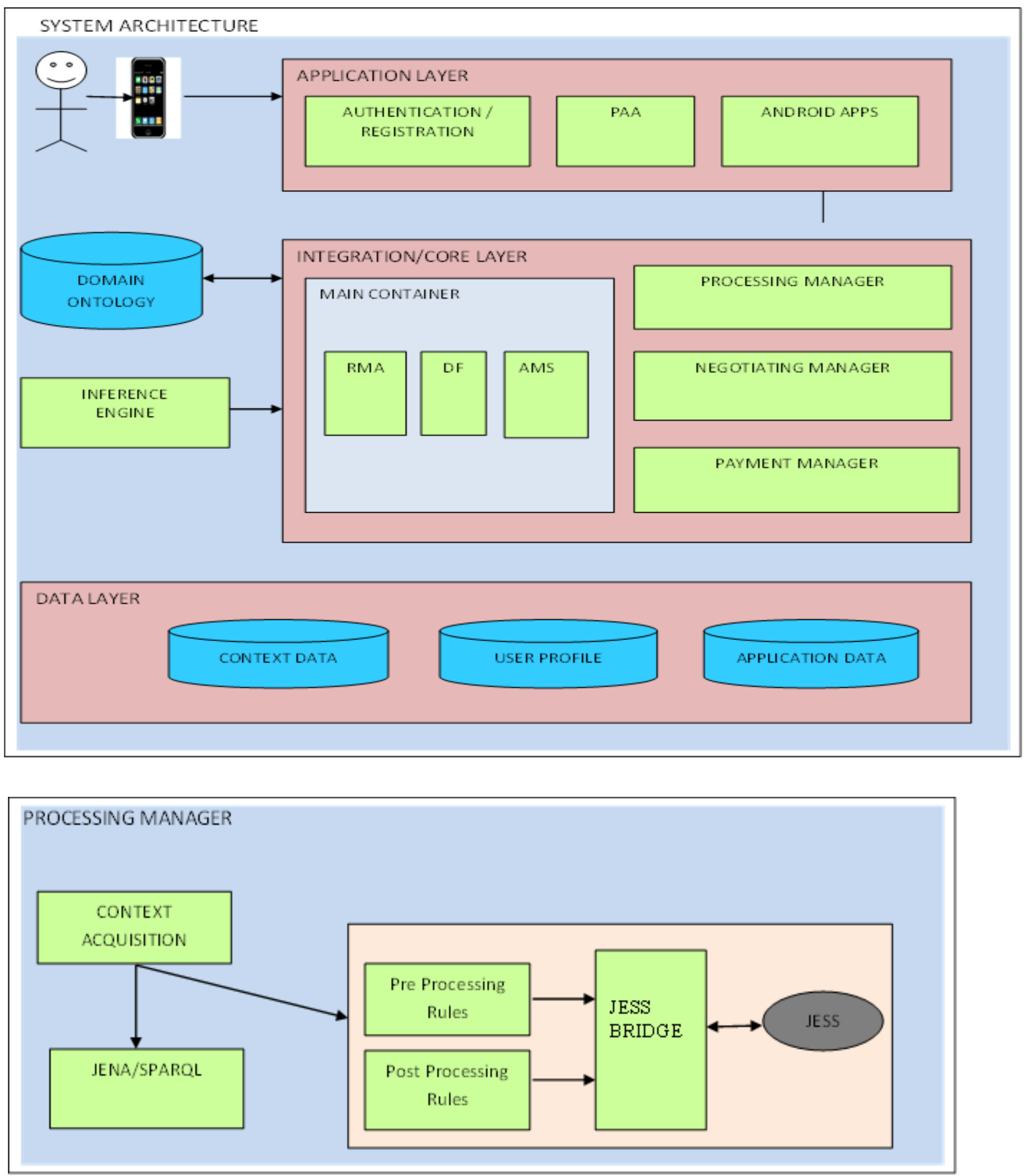

Fig. 2 Processing Manager 


\subsubsection{Negotiating Manager}

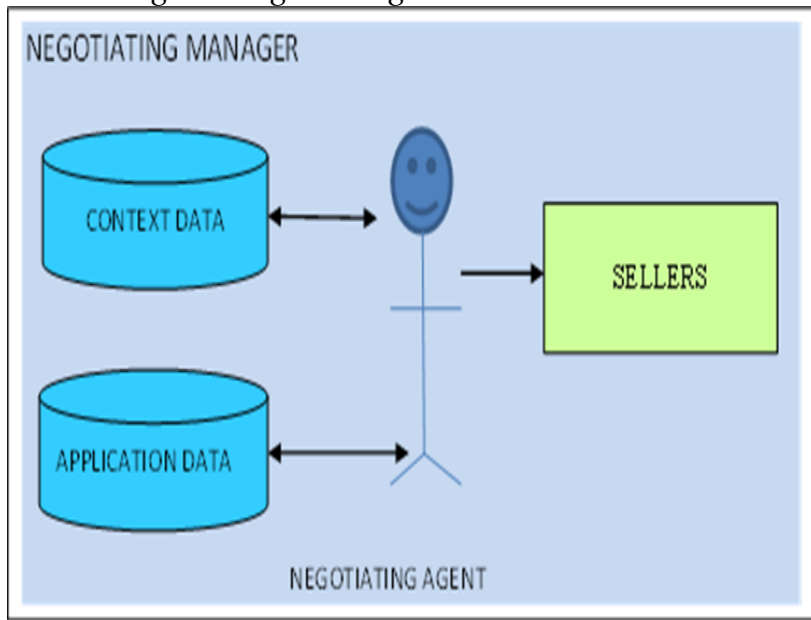

Fig.3 Negotiating Agent

The task of the Negotiating Manager as shown in Fig. 3 is to choose the best sellers based on the Buyers requirement, User preferences and contextual information. The Seller information is then stored in the Application DB and communicated to the Processing Manager.

\subsubsection{Payment Manager}

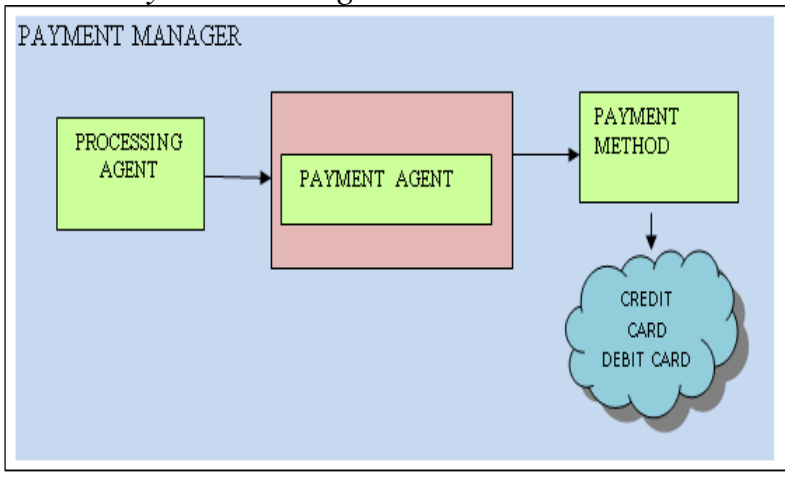

Fig.4 Payment Manager

After the User is satisfied with the product and the seller, he/she initiates the process of completing the Order via PAA to Processing Manager. The Payment manager on receipt of request from Processing Manager uses the Payment method agent to communicate to Merchant bank to complete the payment based on the payment info be it credit card, direct debit etc and informs the Processing Agent about failure or success of the transaction. Internal structure of Processing Manager is shown in Fig. 4

\subsection{Data Layer}

The Data Layer stores persistent data of the iShopper application. It mainly contains User Profile and information on product and services bought by them. The Data Layer is accessed and updated by the sub systems within the Integration/Core Layer. The Archiving module within the Data Layer is responsible for periodically archiving old transaction data..

\section{SYSTEM EVALUATION AND TESTING}

.System is evaluated and tested in three modules

\subsection{Data Set Testing and Evaluation}

The iShopper Application was tested and evaluated against different data-set that is stored in MySQL DB. The data-set in form of objects are stored in MySQL as tables.

- The first data-set stored are related to Customer Profiles and other details like name details, phone details, address details, login details etc.

- The second data-set are related to Sellers like contacts, Phone details, address etc

- The third data-set are related to goods like sellers characteristic, price and sellers sale condition

- The fourth data-set are related to Orders and Order details which are stored as XML

- The fifth data-set are related to Customers payment details like Credit Card, Debit Card etc.

Table 1. Customer Profile Table

\begin{tabular}{|c|c|c|c|c|c|}
\hline uid & firstname & lastname & email & salt & encrypted password \\
\hline 10 & rajesh & shama & 12au97@gmail.com & eb47379bb8 & 8 baEMrXYk7wz266NawKo 76a7... \\
\hline 29 & rajesh & sha & juyal_rajesh@yahoo.com & ee65654dfo & 3dKNiNH/5a $7 w z Z 556 k$ kmgD... \\
\hline 39 & rajesh & shama & juyal.rajesh@gmail.com & $1 e b 904 b 6 \mathrm{~d} 0$ & 0 DcdKyAMpTTz 1 hX_SW \\
\hline 41 & richa & gairola & richagairola@gmail.com & $a 8 a 7 c 57675$ & 5 veKmfrpbRKbHh+6sg06wMH2... \\
\hline 42 & Richa & Shama & richagairola@yahoo.com & $5 f d 7 e 33 e 01$ & jc2UnDSGJF6HOHpz3W5d7M... \\
\hline 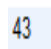 & vidya & raj & vidya_rajh & e18194 & 6 AGRdA1lkEghJ72Y28jROCkz.. \\
\hline
\end{tabular}

Table 2. Address Table

\begin{tabular}{|c|c|c|c|c|c|c|}
\hline address_d & address_ype & addeessl & addess? & ipcode & dity & state \\
\hline 1 & Shipping & 1001. Ariana Raneja Acoopolis & S Deonar pada rood, deonar & 400088 & Mumbo & i Mahrarshtia Inda \\
\hline 2 & Shipping & 1203-Sajsanskrar Complex & Chembur & 400086 & Mumba & Naharastra India \\
\hline 3 & Näiling & B.4shakt ingagr & Dadar & 400011 & Mumbo & Malarastira Inda \\
\hline 4 & Maling & A.117new chaw & Matim & 400033 & Mumbe & i Mahrachttra India \\
\hline 5 & Maling & D.402 Samdit naggar & Ghatkopar & 400086 & Mumbo & Mabrasestra Inda \\
\hline 6 & Maling & thlak nagar & Chembur & 400088 & Mumba & i Maharashtra India \\
\hline
\end{tabular}

Table 3. Seller Information Table

\begin{tabular}{llllll}
\hline sellerid & name & contactname & email & fxedno & mobileno1 \\
\hline 2340 & geniusit & afroos & geniusit@gmail.com & 00912266778856 & 00917776894444 \\
3240 & zoomsquare & zuber & zoomsquare@gmail.com & 00912224489345 & 009199678934093 \\
6780 & dealsquare & vikram & dealsquare@gmail.com & 00912266677799 & 00919967000001 \\
11920 & techiehome & ravi & techihome@yahoo.com & 00912224445676 & 009199678945678 \\
12240 & buyingidea & naresh & buyingidea@redifmail.com & 00912226663333 & 00919821022222 \\
12450 & dealteam & shankar & dealteam@gmail.com & 00912225558909 & 00918967000023 \\
75150 & shoppingwopping & akshat & shoppingwopping@yahoo.com & 00912266778899 & 00918967896789
\end{tabular}

Table 4. Goods Table

\begin{tabular}{llllll}
\hline Sony_Cybershot_DSC_WX300 Cameras & 3240 & 19950 & & $>20000$ & 8 \\
Sony_Cybershot_DSC_WX300 Cameras & 11920 & 19950 & Amex & & \\
Sony_Cybershot_DSC_WX300 Cameras & 12450 & 19950 & Master & & \\
Sony_Cybershot_DSC_WX300 Cameras & 2340 & 19950 & & & \\
Sony_Cyber-shot_DSC_WX300 Cameras & 6780 & 19950 & & $>30000$ & 3 \\
Sony_Cybershot_DSC_WX300 Cameras & 75150 & 19950 & Visa & $>10000$ & 5 \\
Sony_Cyber-shot_DSC_WX300 Cameras & 12240 & 19950 & & & \\
\hline
\end{tabular}




\section{Table 5. Order Transaction Table}

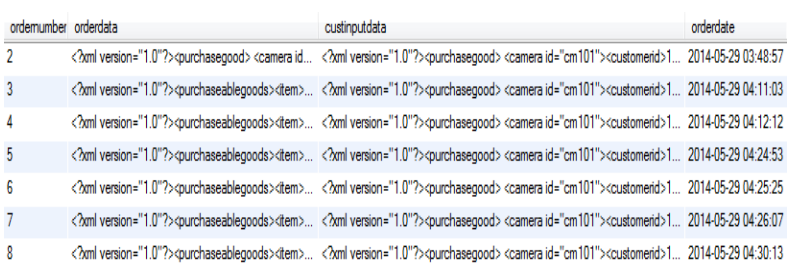

Table 6. Customer Payment Information Table

\begin{tabular}{llll|l|ll} 
id & customer_d & card_type & card_no & cvv & expiry & card_limit \\
\hline 1 & 1001 & Visa & 4778233719989988 & 121 & $06 / 30$ & 100000 \\
2 & 1001 & Master & 4776888976560989 & 141 & $06 / 30$ & 100000 \\
3 & 13 & Visa & 4778233719989101 & 345 & $06 / 30$ & 100000 \\
4 & 13 & Master & 4567801078453567 & 556 & $06 / 30$ & 100000 \\
5 & 1003 & Visa & 4778233719989101 & 789 & $06 / 30$ & 100000 \\
6 & 1003 & Master & 4444029998090288 & 789 & $06 / 30$ & 100000
\end{tabular}

\subsection{Domain Ontology and Evaluation}

The iShopper Application was tested and evaluated against Camera Ontology extensively more than 450 test case where executed. The Camera Domain Ontology was created using Protégé 4.3 and was accessed using Jena/Sparql through the Integration/Core layer. iShopper Product Ontology is shown in Fig.11

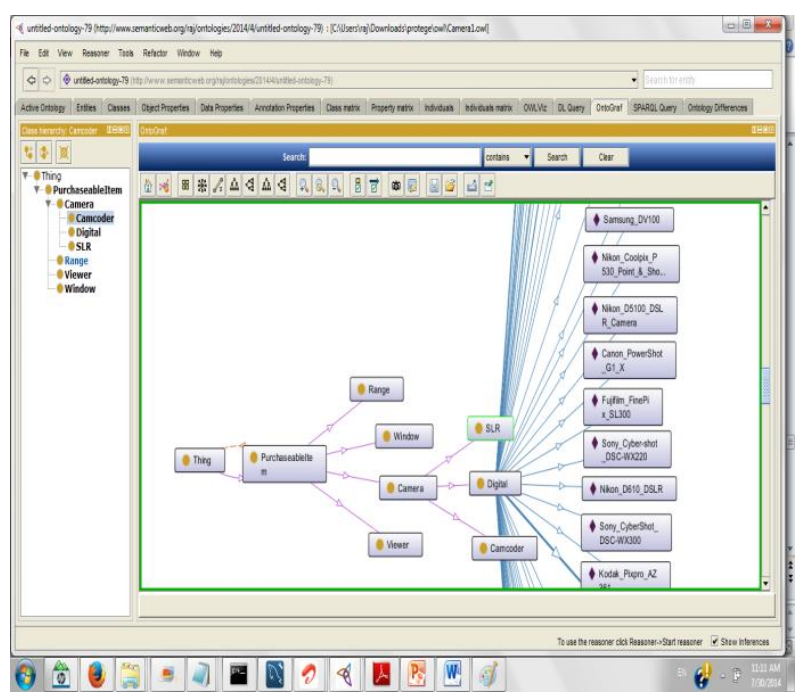

Fig.5 iShopper Product Ontology

\subsection{Application Evaluation and Testing}

After the Customer successfully logging to iShopper application the Customer is ready for purchasing hard goods such as Camera, Camcorder, and Digital content such music, videos, news, ring tones, online game etc.

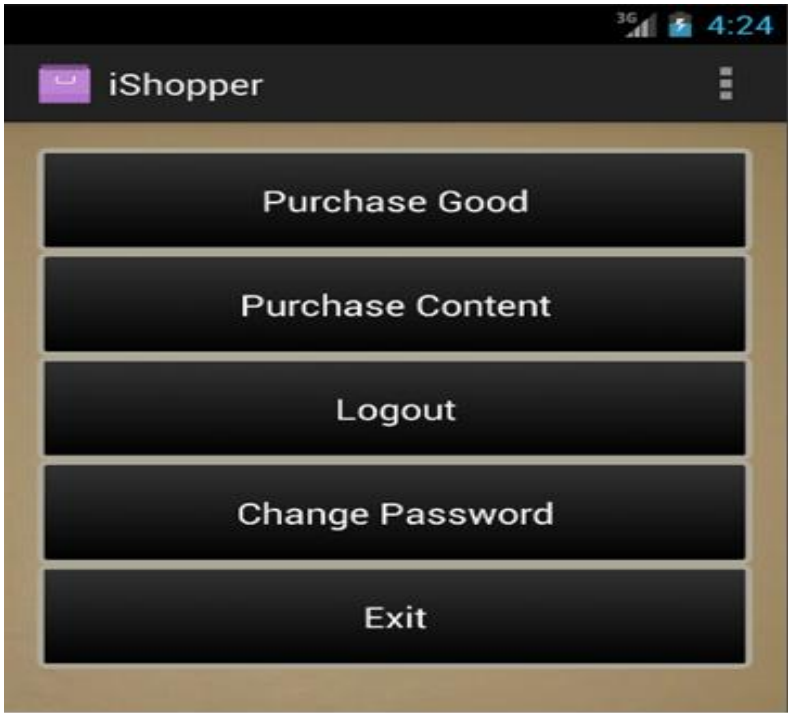

Fig. 6 iShopper Main Screen

\subsubsection{Use Case1}

The User has not researched which Camera he wants to buy and wants the PAA to provide him the best camera option available in the market based on his preferences and requirements. The User enters his requirement on the iShopper GUI installed on the Users Mobile Phone. There are mandatory and optional set of inputs which required by the Application to work effectively and accurately. Some of the mandatory sets of inputs are Price Range and the Purpose or Use of camera. There are other optional set of inputs like Brand, Colour etc which could be additionally entered by the User for filtering his requirement for Camera.

The PAA passes the collected information to the Integration/Core layer which processes and selects the best Camera and best Seller based on the User preferences, requirement, and contextual data. It will communicate details to the User via email and SMS. The User on satisfaction could $\log$ on to iShopper application and complete his order processing.

\subsubsection{Use Case 2}

The User has already researched and come to the conclusion which Camera has to be bought and wants the iShopper to negotiate on his behalf and find the best Seller based on his preferences and contextual data. The PAA passes the collected information to the Integration/Core layer which selects the best Seller based on the User preferences and contextual data. It will communicate details to the User via email and SMS. The User on satisfaction could log on to iShopper application and complete his order processing. 


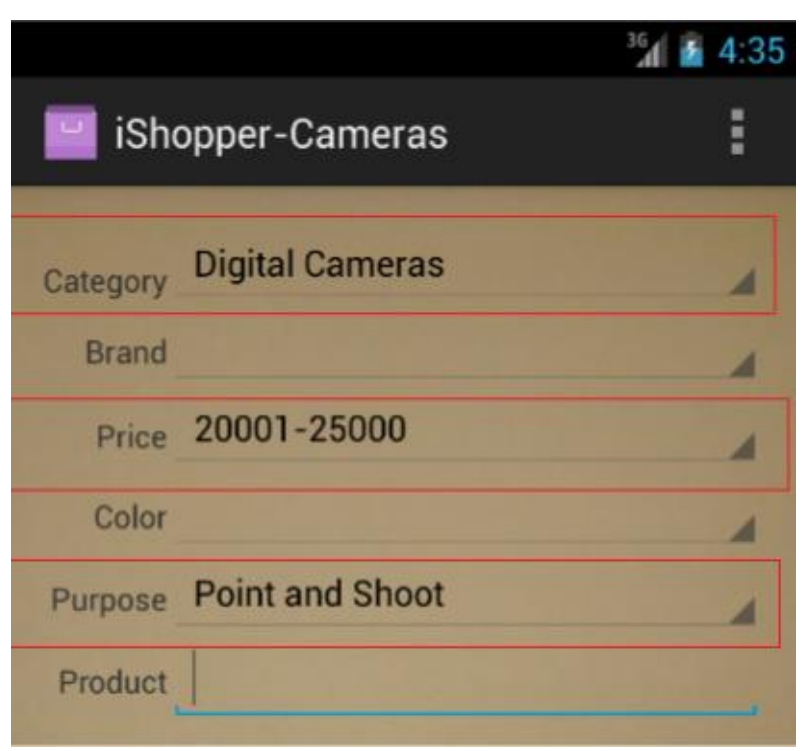

Fig. 7 Use Case1

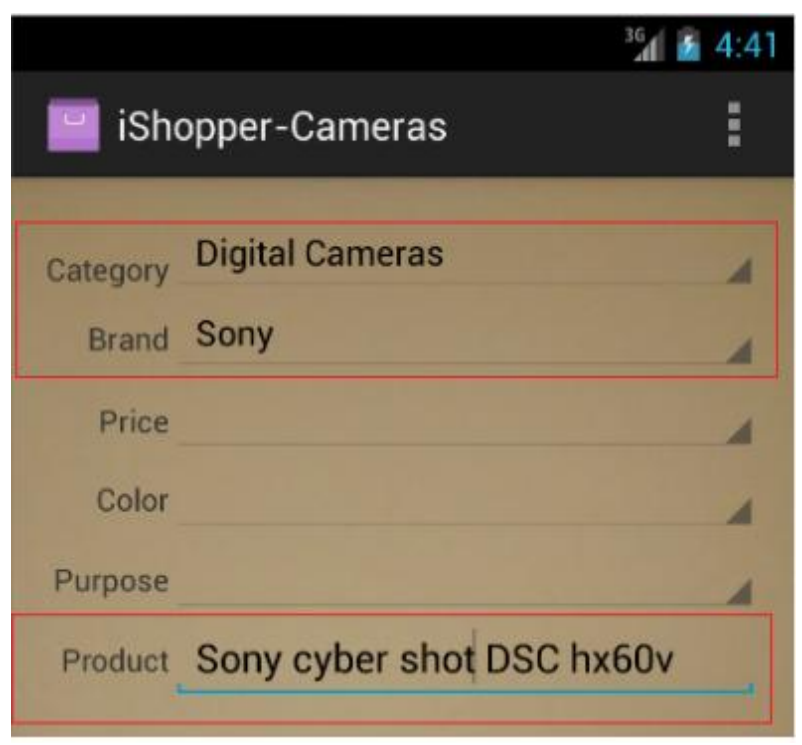

Fig.8. Use Case 2

\subsection{XML Evaluation and Verification}

The XML output generated via iShoppper Application contains the complete details of the Order. XML elements and its value are carefully verified to check the accuracy of the System. In the given XML the user has asked for a Digital Camera in the range of INR20001-25000 and there are 5 cameras which meet his requirements. The Systems after applying pre-processing Jess rule selects Sony_Cybershot_DSC_HX60V as best possible Camera which meets the Customers requirement. Similarly from list of 14 sellers selling the Sony_Cyber-shot_DSC_HX60V the System successfully select the best seller shoppingwopping which met the Customers' requirements .As seen in XML the maximum retail price for the Camera was INR 22500 indicated by the Price tag whereas the Seller offered the same Camera for INR 17100 indicated by Seller Price tag.

$<$ ?xml version="1.0" encoding="UTF-8" standalone="no"?>

$<$ PurchaseOrder $><\mid$ tems $><\mid$ tem $><$ Name $>$ Sony_CyberShot_DS C_HX60V $<$ /Name $><$ Pixel $>20.1</$ Pixel $><$ Zoom $>30.0</$ Zoom $><$
Rating $>5.0<$ Rating $><$ Warranty $>2.0<$ Warranty $><$ Battery $>$ Lb $<$ Battery $><$ Brand $>$ Sony $<$ /Brand $><$ Price $>22500.0<$ /Price $><$ Qu antity $>1</$ Quantity $><$ Seller $><$ Sellerld $>75150<$ Sellerld $><$ Selle rPrice $>17100.0<$ SellerPrice $><$ SellerName $>$ shoppingwopping $<$ SellerName $><$ SellerContact $>$ akshat $<$ /SellerContact $><$ Seller Email $>$ shoppingwopping@yahoo.com $</$ SellerEmail $><$ SellerFi xedNumber $>912266778899</$ SellerFixedNumber $><$ SellerMo bileNumber $1>918967896789</$ SellerMobileNumber $1><$ Seller MobileNumber $2>918967898989</$ SellerMobileNumber $2></ S$ eller $></$ Item $></$ Items $><$ Customer $><$ Customerld $>1001<$ /Cust omerld $><$ CustomerName $>$ RajeshSharma $<$ /CustomerName $><$ CustomerEmail>rajesh.juyal@gmail.com $<$ /CustomerEmail $><C$ ustomerFixedNumber $>009124449456<$ /CustomerFixedNumb er $><$ CustomerMobileNumber $>00919967378909</$ Customer MobileNumber $><$ /Customer $><$ OrderNumber $>429</$ OrderNu mber $><$ OrderDate $>13 / 07 / 2014</$ OrderDate $><$ PaymentInfo $>$ $<$ Name $>$ RajeshSharma $<$ /Name $><$ Amount $>17100.0<$ /Amount $><$ Type $>$ Visa $</$ Type $><$ CardNumber $>4778233719989988</$ Car dNumber $><$ CVV $>121</$ CVV $><$ Expiry $>06 / 18</$ Expiry $></$ Payme ntInfo $><$ Address $><$ Name $>$ RajeshSharma $</$ Name $><$ AddressTy pe $>$ Shipping $<$ /AddressType $><$ Street $>1001$ ArianaRahejaAcrop olis,Deonarpadaroad,deonar $</$ Street $><$ City $>$ Mumbai $</$ City $><$ State $>$ Maharashtra $<$ /State $><$ Zip $>400088</$ Zip $><$ Country $>$ Indi $\mathrm{a}</$ Country $><$ /Address $><$ /PurchaseOrder $>$

\section{CONCLUSION AND FUTURE WORK}

Customers are often confused as which product to buy from plethora of available offline and online product options. Searching both offline and online shops for best product could be tedious and time consuming. Even after the Customer has zeroed down a product, he is unsure whether he has chosen the right seller, paid right price, chosen best warranty and so forth. The iShopper Application is designed and implemented to provide customers with solution for the above mentioned issues. It acts as both ordering systems as well as recommending system, which intelligently tells Customers which product and which seller best matches their requirement. The Customer then has the option either to proceed with the Order or just use the details as recommendation. The iShopper application is built on highly scalable and robust framework; new configurable rules can be introduced within the System with minimum implementation. The iShopper application was tested extensively and the results were very promising, the System accurately predicted results based on the rules and data available within the System.

Currently iShopper Application supports only android operated phones; however we would like to extend to all types of Phones. Further we would also like to enhance the Cosmetic layer of Application by making our Graphical User Interface more attractive and user friendly. Also we intend to improve the accuracy of the system by getting more inputs from customers and industry experts and using them to configure more intricate inference rules.

\section{ACKNOWLEDGMENTS}

We would like to thank department of Information Technology of Vivekananda Education Society's Institute of Technology (VESIT) for providing us opportunity to carry out our work

\section{REFERENCES}

[1] Leila Abedi1, Mohammadali Nematbakhsh2 and Abbas Abdolmaleki, A model for context aware mobile banking, in proceedings. Journal of Theoretical and 
Applied Electronic Commerce Research, volume 7 issue 3, 2012.

[2] Guanling Chen and David Kotz, A Survey of ContextAware Mobile Computing Research, Dartmouth Computer Science Technical Report TR2000-38

[3] J. Lukkari, J. Korhonen, and T. Ojala, Smart restaurant mobile payments in context-aware environment, in Proceedings 6th International Conference on Electronic Commerce, Delft, 2004, pp. 575-582.

[4] F. Bormann, S. Flake, J. Tacken, and C. Zoth, Thirdparty-initiated context-aware real-time charging and billing on an open SOA platform, in Proceedings 22nd International Conference on Advanced Information Networking and Applications, Okinawa, 2008, pp. 13751380 .

[5] Oh Byung Kwon and Norman Sadeh, Applying casebased reasoning and multi-agent intelligent system to context-aware comparative shopping, Decision Support System 37 (2004), pp 199-213.

[6] A. Dey and G. Abowd, Towards a better understanding of context and context-awareness, in Proceedings the 1st international symposium on Handheld and Ubiquitous Computing, Karlsruhe, 1999, pp. 304-307.

[7] J. Bush, J. Irvine, and J. Dunlop, Personal assistant agent and content manager for ubiquitous services, in Proceedings 3rd International Symposium on Wireless Communication Systems, Valencia, 2006, pp. 169-173.

[8] J. Dunlop, The concept of a personal distributed environment for wireless service delivery, Wireless Personal Communications, vol. 42, no. 3,2007, pp. 431444.
[9] S. De, A. Attou, and K. Moessner. Service and content presentation in ubiquitous environments. The University of Surrey. [Online] Available: http://info.ee.survey.ac.uk/CCSR/EuroSSC/2009/poster/ Dec09_EuroSSC.pdf, August 2010.

[10] L. Ning. (2010, August) The MVCE knowledge-based content and service adaptation management framework. Mobile Virtual Center of Excellence. [Online]. Available: www.mobilevce.com.

[11] O. Roozmand, M. A. Nematbakhsh, and A. Baraani; An electronic marketplace based on reputation and learning, Journal of theoretical and applied electronic commerce research, vol. 2 , no. 1,2007 , pp. 1-17.

[12] D. Suparna, and K. Moessner, Device and service descriptions in personal distributed environments, in Proceedings 2nd International Conference on Digital Information Management, Lyon, 2007, pp. 695-700.

[13] M. Shahidi, A. Attou, and H. Aghvami, Content adaptation: requirements and architecture, in Proceedings $10^{\text {th I }}$ nternational Conference on Information Integration and Web-based Applications and Services, Linz, 2008, pp.626-629.

[14] H. Tarus, J. Bush, J. Irvine, and J. Dunlop, Multi-agent mediated electronic-marketplace for adaptation services, in Proceedings of the Fifth IEEE Consumer Communications \& Networking Conference, Las Vegas, 2008, pp.861-862

[15] J. Weijin and Y. Lina, Research on MAS behavior and paradigm learning-based evolutionary method and its application in E-commerce, in Proceedings of the International Symposium on Computer Communication Control and Automation, Tainan, 2010, pp. 458-461 